\title{
Electrocardiographic prediction of coronary artery patency after thrombolytic treatment in acute myocardial infarction: use of the ST segment as a non-invasive marker
}

\author{
K J HOGG, R S HORNUNG, C A HOWIE, N HOCKINGS, F G DUNN, W S HILLIS \\ From the Departments of Cardiology and Materia Medica, University of Glasgow, Stobhill General Hospital, \\ Glasgow
}

SUMMARY The predictive value of the measurement of changes in ST segment elevation was assessed as a non-invasive marker of coronary artery reperfusion after thrombolytic treatment. Forty five patients with acute myocardial infarction (23 anterior, 22 inferior) of less than six hours' duration were given thrombolytic treatment by either the intravenous $(n=28)$ or the intracoronary route $(n=17)$. A proportional value for the shift in ST segment, termed the fractional change, was calculated both from 12 lead electrocardiograms and from the Holter tape for each patient. Coronary artery patency in an initial group of 22 patients (training group) was associated with a fractional change value of $\geqslant 0.5(100 \%$ specific, $88 \%$ sensitive by Holter analysis; $100 \%$ specific, $94 \%$ sensitive by 12 lead electrocardiogram). This rule performed well when it was applied to a test group of 17 patients (100\% specific, $93 \%$ sensitive by Holter analysis; and $67 \%$ specific, $93 \%$ sensitive by 12 lead electrocardiogram). Linear discriminant analysis was then used to determine which features gave the best separation of those in whom there was reperfusion and those in whom there was not. This gave $100 \%$ specificity and $100 \%$ sensitivity when applied to the training group for either the 12 lead electrocardiogram or Holter monitoring. When it was applied to the test group, the sensitivity was maintained at $100 \%$, but the specificity dropped to $33 \%$ irrespective of whether the basis of the test was Holter monitoring or the 12 lead electrocardiogram.

These results suggest that a fractional change of $\geqslant 0.5$ calculated from a single lead showing myocardial injury is a useful non-invasive marker of reperfusion. The technique can be applied to either 12 lead electrocardiograms or Holter monitoring. The use of a more complex classification increased the sensitivity of the test at the expense of its specificity.

Thrombolytic agents are increasingly used to treat patients with acute myocardial infarction. The immediate therapeutic objective is to obtain coronary artery reperfusion, which can only be confirmed definitely by coronary angiography. This invasive technique carries an inherent risk and is limited to major cardiac centres with facilities for cardiac catheterisation. Initially, administration of streptokinase required angiography for selective coronary artery infusions, ${ }^{1}$ but subsequently intravenous

Requests for reprints to Dr K J Hogg, Department of Materia Medica, University of Glasgow, Stobhill General Hospital, Glasgow G21 3UW.

Accepted for publication 16 June 1988 infusions of streptokinase were shown to be effective. $^{23}$ More fibrin specific agents, such as anisoylated streptokinase plasminogen activator complex (APSAC) 4 and tissue plasminogen activator (tPA), ${ }^{5}$ are also effective when given intravenously. The feasibility of giving thrombolytic agents intravenously to large numbers of patients was established by the recent GISSI study ${ }^{6}$ and ISIS 2 study. ${ }^{7}$ Emergency coronary angiography is not now needed for the administration of thrombolytic treatment, but there is a need for non-invasive physiological markers to identify coronary artery reperfusion.

The electrocardiogram is widely used in the clinical diagnosis of acute myocardial infarction and the 
time course of changes in the ST segment is well known. Analysis of the reduction in ST segment elevation, recorded by epicardial and precordial electrodes, was used to reflect coronary artery reperfusion in animal models, ${ }^{8}$ and surface mapping techniques were applied during clinical thrombolysis. ${ }^{9}$

We have studied the ST segment changes occurring in patients during thrombolytic treatment with a view to devising a simple, sensitive, widely applicable technique to detect successful reperfusion noninvasively.

\section{Patients and methods}

We studied 45 patients (age range, 39-75, mean 58.5) with 23 anterior infarcts (in 18 men and five women) and 22 inferior infarcts (in 16 men and six women) who were admitted a mean (SD) of $2.7(1.6)$ hours after the onset of pain. Patients were treated with thrombolysis if they had: (a) cardiac pain lasting at least 30 minutes but less than six hours by the time of admission to the coronary care unit; and (b) confirmatory electrocardiographic evidence of myocardial infarction (ST elevation $\geqslant 1 \mathrm{~mm}$ in at least two standard limb leads or $2 \mathrm{~mm}$ ST elevation in at least two precordial leads). Patients were not treated with thrombolysis if they had (a) a history of bleeding diathesis, $(b)$ a recent cerebrovascular accident, $(c)$ peptic ulceration, or (d) severe renal or hepatic disease. Informed consent for administration of thrombolytic agents and for coronary arteriography was obtained in both groups.

The patients were admitted to the coronary care unit of Stobhill General Hospital and thrombolytic treatment was given according to the current coronary care protocol. Seventeen patients were given streptokinase by the intracoronary route, and 28 were given intravenous anisoylated plasminogen streptokinase activator complex (APSAC) (Eminase). The division of these patients into these groups depended on the current treatment and was not affected by the clinical severity of the illness on admission.

INTRACORONARY GROUP

Emergency coronary arteriography was performed (Judkins' technique) within the coronary care unit with an image intensification system (Siemens $2 \mathrm{H}$ $2 \mathrm{~N}$ ) and a high quality video tape recorder for permanent data storage. ${ }^{10}$ The occluded infarct related artery was identified and streptokinase 250 500000 units was infused selectively or subselectively over $30-45$ minutes. The presence or absence of coronary artery reperfusion was determined visually at the end of the infusion.

INTRAVENOUS GROUP

Anisoylated plasminogen streptokinase complex
(APSAC) (30 mg) was given over five minutes Angiography was not performed before treatment but coronary artery patency was confirmed bo: arteriography performed at 90 minutes after treat $t^{5}$ ment. Angiography was delayed in some subjects until haemodynamic or electrical stability was achieved.

\section{ELECTROCARDIOGRAPHIC ANALYSIS \\ Holter monitoring}

On admission to the coronary care unit, 24 hou continuous electrocardiographic monitoring was. started with an Oxford Medilog II system. Record $\frac{}{5}$ ing electrodes were placed in a modified V5 position? The tapes were analysed with a Reynolds Medicas Pathfinder by an ST segment trend analysis. The ST segment elevation was measured as a pretreatmenk control value $\left(M_{1}\right)$ and a second measurement $\left(M_{2}\right)$ was made two hours after intracoronary treatmen? and three hours after intravenous thrombolytic treat ment. The value after treatment was calculated as proportion of the control value and this index was called the fractional change (fig 1). With complete reversal of the ST segment elevation the fractionato change is one or a number near to one (fig la). If thẹ ST segments remain elevated to the same degree the fractional change is around zero. When the ST segment elevation increases because of infarct exten $\frac{5}{\partial}$ sion, the fractional change is negative (fig $1 \mathrm{~b}$ ).

\section{Twelve lead electrocardiograms}

Conventional 12 lead electrocardiography was perợ formed with a Hewlett-Packard Electrocardiograph 4700A on admission and at a mean (SD) of 302 (141 min after admission. The positions of electrodes of the chest were marked to ensure reproducibility. ST segment elevation was measured at the $J$ point, and the lead showing the most ST segment elevation was taken for serial analysis. The fractional change waş. calculated in the same way as it was for Holte? monitoring. In both studies, the electrocardiograms were read blindly without knowledge of the angiogram results.

STATISTICAL ANALYSIS

Linear discrimination was performed on an ICI 2980 main frame computer (program BMDP (P7M) ${ }^{11}$ The patients were randomly subdivided by a randory number generator into two groups: one a trainiris group of 22 for devising a rule and a second test groug of 17 for checking it. The sensitivity measures th ability of a test to detect genuine instances of reperfusion and was expressed as the number of cases of reperfusion detected by the non-invasive test as 9 percentage of angiographically confirmed repexक fusions. Specificity measures the ability of a test tof 

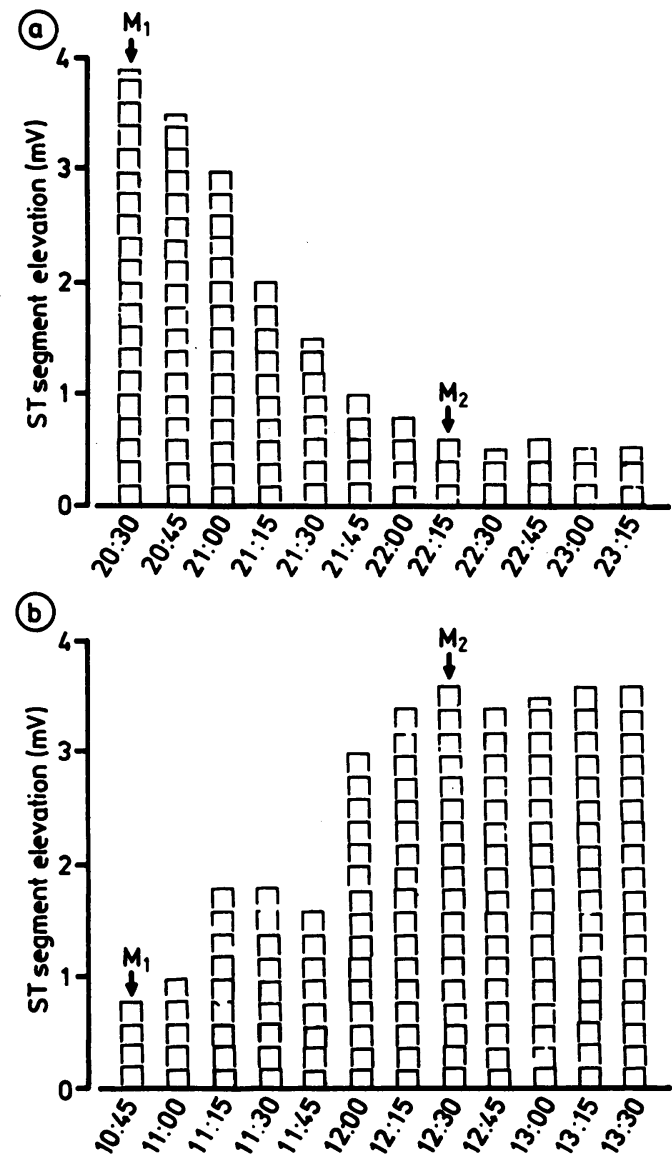

Fig 1 Typical examples of $S T$ segment analysis from Holter monitor recordings in patients treated with intracoronary thrombolysis. (a) A successful reperfusion. (b) No reperfusion. Measurement $M_{1}$ was made on admission before treatment and $M_{2}$ two hours after treatment. The fractional change was calculated as $\left(M_{1}-M_{2}\right) \div M_{1}$. For (a) the fractional change is $0.85((3.9-0.6) \div 3.9)$ and for (b) it is $-3.5((0.8-3.6) \div 0.8)$.

detect genuine instances in which there has not been reperfusion. It was expressed as the number of true cases of non-reperfusions detected' by the noninvasive test as a percentage of angiographically confirmed cases of non-reperfusion.

\section{Results}

We analysed the electrocardiographic recordings of 39 subjects (six were not analysed). In four patients there was incomplete collection of data because the Holter monitoring tapes were technically inadequate, in one patient a left bundle branch block precluded interpretation of the ST segment, and in another patient the coronary arteriogram was inadequately visualised. The remaining 39 patients were randomly split into a training group $(\mathbf{n}=22)$ and a test group $(n=17)$. Figure 2 shows scatter of the values of fractional changes for these two groups, both for the Holter tapes and the 12 lead electrocardiograms. The arteriographic patency rate of the coronary arteries was high (79.5\%). Because the ST segment elevation is not completely reversed after coronary artery reperfusion, several arbitrary values of ST segment reduction were examined including $0.25,0.5$, and 0.75 . A fractional change of 0.5 seemed to be the most useful value for separating those cases with reperfusion from those without. Six of the total of nine patients without reperfusion showed more ST segment elevation on the second electrocardiogram, resulting in a negative fractional change. In the three in whom the elevation of the ST segment was reduced without angiographic evidence of reperfusion the fractional change was $<0.5$. There were no differences in mean (SD) ST segment elevation on the presentation electrocardiogram between those who had reperfusion $(3.8(2.4) \mathrm{mm})$ and those who did not $(3 \cdot 1(2 \cdot 1) \mathrm{mm})$. The table gives the specificity and sensitivity in both the training and test groups for detecting reperfusion by a fractional change of 0.5 . We then used linear discrimination with a $B M D P$ program to devise a rule that would give maximum discrimination between the two groupsreperfusion and non-reperfusion. This program used the following electrocardiographic variables: fractional change with Holter monitoring (FCH), fractional change with 12 lead electrocardiogram (FC), $M_{1}$ (height of ST segment on admission), and $M_{2}$ (height of ST segment after treatment). We derived the following classification functions for the training group:
(a) Using fractional change with Holter monitoring
Reperfusion $=-3.31+(2.42 \times \mathrm{FCH})+$
$\left(1.45 \times M_{1}\right)-\left(1.36 \times M_{2}\right)$
Non-reperfusion $=-8.48-(4.74 \times \mathrm{FCH})-$
$\left(1.03 \times M_{1}\right)+\left(3.72 \times M_{2}\right)$
(b) Using fractional change
Reperfusion $=-2.80+(1.21 \times \mathrm{FCH})+$ $\left(1.28 \times M_{1}\right)-\left(0.87 \times M_{2}\right)$

Table The specificity of a fractional change of $\geqslant 0.5$ for the detection of reperfusion

\begin{tabular}{|c|c|c|}
\hline ECG variable & Specificity $(\%)$ & Sensitivity (\%) \\
\hline \multicolumn{3}{|c|}{ Fractional change by Holter monitoring } \\
\hline Training group & 100 & $88^{\circ}$ \\
\hline \multicolumn{3}{|c|}{$\begin{array}{c}100 \\
\text { Test group } \quad \text { Fractional change by } 12 \text { lead ECG } 93\end{array}$} \\
\hline Training group & 100 & 94 \\
\hline
\end{tabular}

ECG, electrocardiogram. 
(a) Holter monitor

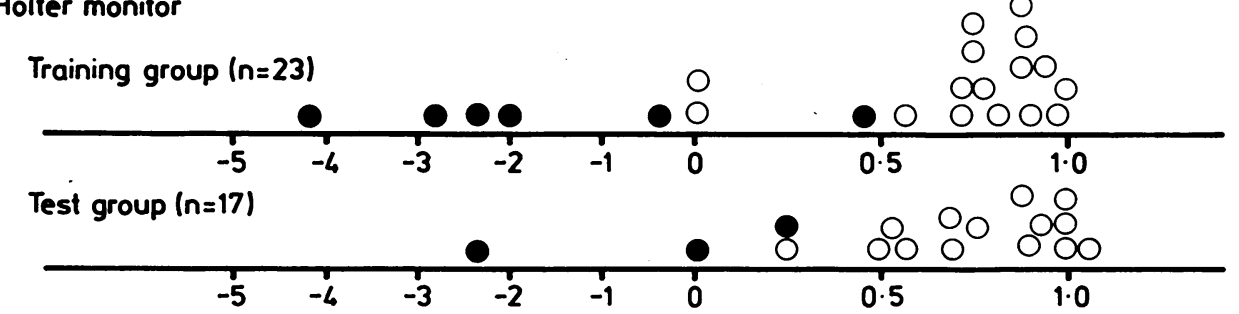

(b) 12 lead ECG

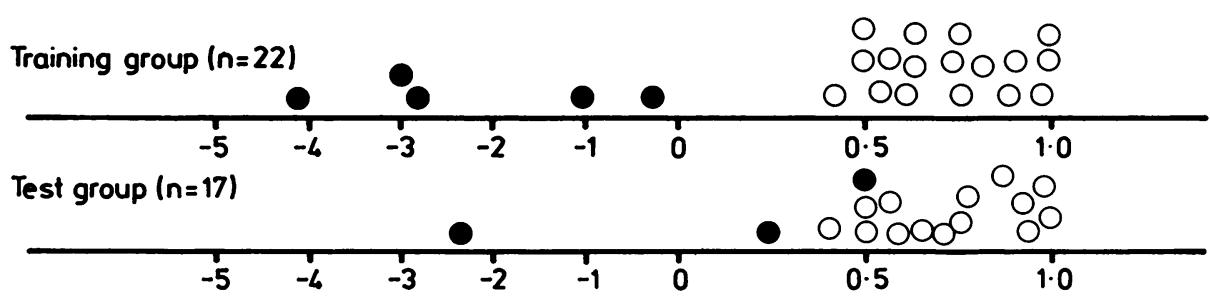

O Reperfusion

- No reperfusion

Fig 2 Scatter diagrams showing fractional changes calculated for Holter monitoring (a) and 12 lead electrocardiograms (b).

Non-reperfusion $=-7.01+(-3.09 \times \mathrm{FCH})-$ $\left(0.57 \times M_{1}\right)+\left(2.64 \times M_{2}\right)$

To use these classification functions, the value of the fractional change (either from 24 hour tapes or 12 lead electrocardiograms) and the height of the ST segment on admission and after treatment must be known. These values were substituted into the formulas for reperfusion and for non-reperfusion; the patient was identified as belonging to the group formula that gave the higher value. For example, a patient with an FCH of 0.65 and $M_{1}=3.5$ and $M_{2}=$ 1.7 , gives values of 1.03 for the reperfusion group and -8.84 for the non-reperfusion group. As 1.03 is $>-8 \cdot 84$, these findings are consistent with reperfusion.

This form of discriminant analysis correctly identified all the patients in the training group (sensitivity $100 \%$, specificity $100 \%$ ). The sensitivity was the same in the test group, but the specificity dropped to $33 \%$. This dramatic fall in specificity is partly attributable to the small numbers in the test group with no reperfusion $(n=3)$ and the fact that only one of these three patients was correctly identified.

\section{Discussion}

These results confirm the usefulness of ST segment analysis as a non-invasive predictor of coronary artery patency after thrombolytic treatment in patients with acute myocardial infarction. This tech- nique can therefore identify patients who may require further pharmacological treatment to preven? reocclusion, or who may require subsequent invasive investigation before coronary angioplasty or bypass surgery for residual arterial stenosis. A fractiona $\bar{\Phi}$ change of $\geqslant 0.5$ was more specific but not as sensitive as the more complex classification functions derived from linear discrimination. The fractional change derived from the Holter monitor was more specifi but not quite as sensitive as that calculated from the 12 lead electrocardiogram. The lack of sensitivity was the result of a fractional change of zero in two patients. in whom angiography confirmed reperfusion. These two patients had inferior myocardial infarction, an it may be that the standard lead positions used are les? effective in showing myocardial injury in the inferios wall of the heart. In addition, the differences in. sensitivity and specificity between the Holter tape and the 12 lead electrocardiogram may to some extent reflect the differences in times over which the change was measured: two or three hours with the $\mathbf{2 4}$ houro tape and a mean of five hours with the 12 lead electrocardiogram. This was the earliest period thate 12 lead electrocardiogram was performed after the patient's return to the coronary care unit after angiography. Measurement of the fractional change over a longer period may have produced fractionat changes that were nearer to 1 , because the natural sequential decline in the elevation of the ST segmem would have contributed to this greater value. If 
theory, extending the time over which the electrocardiograms were performed may have reduced both the sensitivity and specificity and have overestimated the reperfusion rate. This, however, does not seem to be a problem in our study, but it suggests that the earlier determination of the fractional change is more appropriate.

After the work of Pardee, who recognised that ST segment elevation was a marker of coronary occlusion, ${ }^{12}$ many experimental and clinical studies have attempted to use this functional electrocardiographic marker as an index of ischaemic injury and to assess pharmacological interventions designed to limit infarct size. ${ }^{1314}$ Although previous studies have shown a rapid sequential change in ST segments after successful thrombolysis ${ }^{39}$ the usefulness of these changes in identifying reperfusion noninvasively was not studied. A disadvantage of this technique is that the ST segment may be altered by factors other than coronary occlusion such as pericarditis $^{15}$ or metabolic abnormalities including hyperkalaemia. ${ }^{16}$ The presence of interventricular conduction defects, including right or left bundle branch block, or the use of a ventricular pacemaker will also invalidate this technique. ${ }^{17}$

The complex precordial mapping systems with on line computer analysis used by von Essen et al to determine ST segment changes after thrombolysis ${ }^{9}$ is technically difficult, expensive, and limited to a few specialised cardiac centres. Madias et al suggested that the 12 lead electrocardiograph was more useful than anterior precordial mapping in diagnosing and following serial changes in patients with inferior myocardial infarcts. ${ }^{18}$ Leinbach et al further confirmed that both the rate and degree of ST segment fall could be followed accurately by single leads that reflected the zone of maximum electrocardiographic injury. ${ }^{19} \mathrm{We}$ too were successful when we used a suitable precordial lead to show ST elevation in either a Holter tape or the standard 12 lead electrocardiogram.

Continuous electrocardiographic monitoring has the advantage of incorporating a computerised assessment of the ST segment change although it is more complex and time consuming to analyse. The 12 lead electrocardiogram is widely available, inexpensive, and reproducible and can be used quickly at the bedside to reach decisions regarding further management including drug treatment.

The best method for clinical use depends on the specificity and sensitivity of the test. These may change depending on the purpose for which they are being used. Reocclusion has been reported in up to $30 \%$ of patients after successful coronary artery reperfusion. Pharmacological regimens have recently been developed to prevent reocclusion. A test that is highly sensitive in detecting reperfusion will be of value in deciding which patients require follow on treatment. Comparison of reperfusion rates after administration of different thrombolytic agents would require a high specificity. Though we tried to validate our rule in training and test groups, both groups were small and this gives wide errors in estimation of confidence intervals for sensitivity and specificity. The test may need to be assessed in much larger groups.

It may be that the high percentage of patients in the non-reperfusion group who showed an increase in ST segment elevation resulting in a negative fractional change reflects reocclusion rather than just non-reperfusion. By definition reocclusion occurs only in vessels that initially have been reperfused; this would result in a biphasic curve on the ST segment printout. When we examined our patients none of them showed such a curve, and, in addition, no patient had recurrence of chest pain to suggest reocclusion. Application of this technique may be useful if studied over a longer period.

In summary, fractional change can be measured from a single lead by Holter monitoring techniques or from 12 lead electrocardiograms, by examining the lead showing most evidence of myocardial injury. It is a useful non-invasive marker of reperfusion, and a value of $\geqslant 0.5$ predicts a patent artery within the time scale of our study. These changes are independent of the agent used and reflect adequate coronary artery perfusion; they may therefore be used to assess the efficacy of other thrombolytic drugs such as tissue plasminogen activator. Use of a more complex classification function increased the sensitivity at the expense of a decline in specificity. The discrimination of the test may be improved by the addition of other variables such as creatine kinase release curves or the presence or absence of reperfusion arrhythmias.

\section{References}

1 Kennedy JW, Ritchie JL, Davis KB, Stadius ML, Maynard C, Fritz JK. The Western Washington randomized trial of intracoronary streptokinase in acute myocardial infarction. $N$ Engl $J$ Med 1985;312:1073-8.

2 Schroder R, Biamino G, von Leitner ER, et al. Intravenous short term infusion of streptokinase in acute myocardial infarction. Circulation 1983;67: 536-48.

3 Anderson JL, Marshal HW, Askins JC, et al. A randomized trial of intravenous and intracoronary streptokinase in patients with acute myocardial infarction. Circulation 1984;70:606-18.

4 Been M, de Bono DP, Muir AL, Boulton FE, Hillis WS, Hornung $R$. Coronary thrombolysis with intravenous anisoylated plasminogen-streptokinase 
complex BRL 26921. Br Heart J 1985;53:253-9.

5 Verstraete M, Brower RW, Collen D, et al. Doubleblind randomised trial of intravenous tissue type plasminogen activator versus placebo in acute myocardial infarction. Lancet 1985;i1:965-9.

6 GISSI Study Group. Effectiveness of intravenous thrombolytic treatment in acute myocardial infarction. Lancet 1986;i:397-401.

7 ISIS Steering Committee. Intravenous streptokinase given within 0-4 hours of onset of myocardial infarction reduced mortality in ISIS 2. Lancet 1987;i:502.

8 Muller JE, Maroko PR, Braunwald E. Evaluation of precordial electrocardiographic mapping as a means of assessing changes in myocardial ischaemic injury. Circulation 1975;52:16-27.

9 von Essen R, Schmidt W, Uebis R, et al. Myocardial infarction and thrombolysis. Electrocardiographic short term and long term results using precordial mapping. Br Heart $J$ 1985;54:6-10.

10 Hillis WS, Jones CR, Been M, Campbell BC, Fulton WFM. Intracoronary thrombolytic therapy performed within a coronary care unit: one year's experience. Scott Med J 1986;31:25-9.

11 Brown MB. Biomedical computer programs. P-series. $B M D P-77$. Berkley, California: University of California Press, 1977.

12 Pardee HEB. An electrocardiographic sign of coronary artery obstruction. Arch Intern Med 1920;26:244.
13 Gold HK, Leinbach RC, Maroko PR. Propranolo ? induced reduction of signs of ischaemic injury during: acute myocardial infarction. Am J Cardiol 1976 38:689-95.

14 Maroko PR, Hillis LD, Muller JE, et al. Favourable effects of hyaluronidase on electrocardiographic evidence of necrosis in patients with acute myocardiaf infarction. $N$ Engl J Med 1977;296:898-903.

15 Thadani U, Chopra MP, Aber CP, Portal RW. Pericar ditis after acute myocardial infarction. $\mathrm{Br} \mathrm{Med} \mathcal{L}$ 1971;i1:135-7.

16 Levine HD, Wanzer SH, Merrill JP. Dialysable currents of injury in potassium intoxication resemW bling acute myocardial infarction or pericarditis. Circulation 1956;13:29-36.

17 Sodi-Pallares D, Cisneros F, Medrano GA, Bisteni A Testelli MR, de Micheli A. Electrocardiographie diagnosis of myocardial infarction in the presence of bundle branch block (right and left), ventriculafr premature beats and Wolff-Parkinson-White syns drome. Prog Cardiovasc Dis 1963; 6:107-136.

18 Madias JE, Venkataraman K, Hood WB. Precordial STO segment mapping 1 . Clinical studies in the coronar care unit. Circulation 1975;52:799-809.

19 Leinbach RC, Gold HK, Harper RW, Buckley MJ, Austen WG. Early intra-aortic balloon pumping for anterior myocardial infarction without shock Circulation 1978;58:204-10. 